Article

\title{
Sign-Periodicity of Traces of Singular Moduli
}

\section{Dohoon Choi ${ }^{1}$, Byungchan Kim ${ }^{2}$ and Subong Lim ${ }^{3, *}$}

${ }^{1}$ School of Liberal Arts and Sciences, Korea Aerospace University, 200-1, Hwajeon-dong, Goyang, Gyeonggi 412-791, Korea; E-Mail: choija@kau.ac.kr

${ }^{2}$ School of Liberal Arts and Institute of Convergence Fundamental Studies, Seoul National University of Science and Technology, 172 Gongreung 2 dong, Nowongu, Seoul 139-743, Korea;

E-Mail: bkim4@seoultech.ac.kr

${ }^{3}$ School of Mathematics, Korea Institute for Advanced Study, Hoegiro 85, Dongdaemun-gu, Seoul 130-722, Korea

* Author to whom correspondence should be addressed; E-Mail: subong@kias.re.kr; Tel.: +82-2-958-3831; Fax.: +82-2-958-3786.

Received: 9 August 2013; in revised form: 9 September 2013 / Accepted: 8 October 2013/ Published: 15 October 2013

\begin{abstract}
Zagier proved that the generating functions of traces of singular values of $J_{m}(z)$ are weight $\frac{3}{2}$ weakly holomorphic modular forms. In this paper we prove that there is the sign-periodicity of traces of singular values of $J_{m}(z)$.
\end{abstract}

Keywords: singular moduli; sign change; circle method

Classification: MSC primary 11F37; secondary 32N10

\section{Introduction and Statement of Results}

Singular moduli are the values of a modular function at the points in the upper half plane $\mathbb{H}$ that satisfy a quadratic equation with rational coefficients. These algebraic numbers play prominent roles in number theory. For example, they generate Hilbert class fields of imaginary quadratic fields and isomorphism classes of elliptic curves with complex multiplication, distinguished by singular moduli.

In his renowned paper [1], D. Zagier displayed an amazing formula, which relates the traces of singular moduli to the Fourier coefficients of a weakly holomorphic modular form of weight $\frac{3}{2}$ and provided new proof of Borcherds' theorem on the infinite product expansions of integer weight modular 
forms on $\mathrm{SL}_{2}(\mathbb{Z})$ with Heegner divisor. In particular, he showed that the trace of the singular moduli for discriminant $-d$ appears as the $d$-th Fourier coefficient of the weakly holomorphic modular form $g_{1}(z)$

$$
g_{1}(z)=\frac{\eta^{2}(z)}{\eta(2 z)} \frac{E_{4}(4 z)}{\eta^{6}(4 z)}=q^{-1}-2+248 q^{3}-492 q^{4}+\cdots
$$

where $\eta(z)=q^{1 / 24} \prod_{n=1}^{\infty}\left(1-q^{n}\right)$ is the Dedekind eta function and $E_{4}(z)=1+240 \sum_{n=1}^{\infty} \sigma_{3}(n) q^{n}$ is the Eisenstein series of weight 4 . This result has motivated numerous studies : Arithmetic property of traces of singular moduli [2-4], modular form grids [4-6], and generalizations [7-9]. Further, sign changes of the Fourier coefficients of modular forms $f(z)$ (especially, when $f(z)$ is a Hecke eigenform) have been extensively studied [10-13]. This is particularly interesting when Fourier coefficients of the modular form encode interesting arithmetic information. For example, the sign-periodicity of certain rank and/or crank differences of integer partitions have been investigated [14-16].

Our aim in this note is to study the sign-periodicity of traces of singular moduli. We prove that the signs of traces of singular moduli of $J(z)$ change periodically by looking at the signs of Fourier coefficients of $g_{1}(z)$. Here, $J(z)$ is the normalized Hauptmodul for $\mathrm{SL}_{2}(\mathbb{Z})$ defined by $J(z)=j(z)-744$ and $j(z)$ is the modular invariant. Actually, we show the sign-periodicity for the traces of singular moduli of $J_{m}(z)$, defined for every non-negative integer $m$ as the unique modular function having the Fourier expansion of the form $q^{-m}+O(q)$.

First we recall the basic notions of the traces of singular moduli following the discussion in [1]. Let $d$ be a positive integer with $d \equiv 0$ or $3(\bmod 4)$. We denote by $\mathcal{Q}_{d}$ the set of positive definite binary quadratic forms $Q=[a, b, c]=a X^{2}+b X Y+c Y^{2}(a, b, c \in \mathbb{Z})$ of discriminant $b^{2}-4 a c=-d$ with the usual action of $\mathrm{SL}_{2}(\mathbb{Z})$. Then the modular trace function $\mathbf{t}_{m}(d)$ is defined by

$$
\mathbf{t}_{m}(d):=\sum_{Q \in \mathcal{Q}_{d} / S L_{2}(\mathbb{Z})} \frac{1}{\omega_{Q}} J_{m}\left(\alpha_{Q}\right)
$$

for all $d$ and all $m \geq 1$, where $\omega_{Q}=\left|\mathrm{SL}_{2}(\mathbb{Z})_{Q}\right|$ and $\alpha_{Q}$ is the unique root of $Q$ in the upper half plane $\mathbb{H}$. Our main result determines the sign of $\mathbf{t}_{m}(d)$.

Theorem 1 With the above notations, we have

$$
\operatorname{sign}\left(\mathbf{t}_{m}(d)\right)= \begin{cases}+ & \text { if } d m^{2} \equiv 0(\bmod 4) \\ - & \text { if } d m^{2} \equiv 3(\bmod 4)\end{cases}
$$

This theorem follows from the result concerning the signs of Fourier coefficients of $g_{1}(z)$ and $\left(\left.g_{1}\right|_{\frac{3}{2}} T\left(m^{2}\right)\right)(z)$, where $\left.\right|_{\frac{3}{2}} T\left(m^{2}\right)$ denotes the action of the $m$ th Hecke operator on $M_{\frac{3}{2}}^{!,+}\left(\Gamma_{0}(4)\right)$. Here, $M_{\frac{3}{2}}^{!,+}\left(\Gamma_{0}(4)\right)$ is the Kohnen plus space of weakly holomorphic modular forms of weight $\frac{3}{2}$ on $\Gamma_{0}(4)$. For any positive integer $m$ let $B_{m}(1, d)$ denote the coefficient of $q^{d}$ in $\left(\left.g_{1}\right|_{\frac{3}{2}} T\left(m^{2}\right)\right)(z)$. Zagier proved the beautiful relation between the modular trace function $\mathbf{t}_{m}(d)$ and the Fourier coefficient $B_{m}(1, d)$ (see Theorem 5 in [1])

$$
\mathbf{t}_{m}(d)=-B_{m}(1, d)
$$

Therefore, the sign-periodicity of $\mathbf{t}_{m}(d)$ in Theorem 1 is an immediate consequence of the following theorem. 
Theorem 2 With the above notations, we have

$$
\operatorname{sign}\left(B_{m}(1, d)\right)= \begin{cases}- & \text { if } d m^{2} \equiv 0(\bmod 4) \\ + & \text { if } d m^{2} \equiv 3(\bmod 4)\end{cases}
$$

Remark 1 By the famous duality result (see Theorem 5 in [1]), the above theorem also says the sign-periodicity of $A_{m}(1, d)$, where $A_{m}(1, d)$ is the 1 st Fourier coefficient of $\left(\left.\left(f_{d}\right)\right|_{\frac{1}{2}} T\left(m^{2}\right)\right)(z)$. Here, $\left.\right|_{\frac{1}{2}} T\left(m^{2}\right)$ denotes the action of the mth Hecke operator on the space $M_{\frac{1}{2}}^{!,+}\left(\Gamma_{0}(4)\right)$ and $f_{d}(z)$ is the unique modular form in $M_{\frac{1}{2}}^{!,+}\left(\Gamma_{0}(4)\right)$ having the Fourier expansion of the form $q^{-d}+O(q)$.

To prove Theorem 2, we obtain an effective estimate of $B_{1}(1, d)$ by employing the circle method. For the sign of $B_{m}(1, d)$ of general $m$, we use the Fourier coefficient formula for the Hecke operator $T\left(m^{2}\right)$. Since the main term in the estimation of $B_{1}(1, d)$ increases exponentially, one expects the sign of $B_{m}(1, d)$ is determined by $B_{1}\left(m^{2} d\right)$ for sufficiently large $d$. The main part of the proof is to find the effective bound for $d$. Then we can verify the sign-periodicity by checking the first few $B_{m}(1, d)$.

\section{Proof of Theorem 2}

We first estimate $B_{1}(1, d)$ with an effective bound by employing the circle method. Before that, we recall basic facts on the circle method. For a series expansion of the form $f(s):=\sum_{n=0}^{\infty} b(n) s^{n}$, by Cauchy's integral formula, we have

$$
b(n)=\frac{1}{2 \pi i} \int_{|s|=r} \frac{f(s)}{s^{n+1}} d s
$$

We will integrate Equation (6) over a circle of radius $r=e^{\frac{-2 \pi}{N^{2}}}:=e^{-2 \pi \rho}$ for a positive integer $N$ to be determined. By following the dissection given in (pp. 115-117 in [14]) or ([17] [Chapter 5]) and setting $z=k(\rho-i \varphi)$ and $\tau=\frac{h+i z}{k}$, we arrive at

$$
b(n)=\sum_{1 \leq k \leq N} \sum_{\substack{0 \leq h \leq k \\(h, k)=1}} e^{\frac{-2 \pi i n h}{k}} \int_{\xi_{h, k}} f(\tau) e^{2 \pi n \rho} e^{-2 \pi i n \varphi} d \varphi
$$

where $\xi_{h, k}=\left[-\theta_{h, k}^{\prime}, \theta_{h, k}^{\prime \prime}\right]$ and

$$
\begin{aligned}
\theta_{h, k}^{\prime} & =\frac{h}{k}-\frac{h_{0}+h}{k_{0}+k} \\
\theta_{h, k}^{\prime \prime} & =\frac{h_{1}+h}{k_{1}+k}-\frac{h}{k}
\end{aligned}
$$

Here, $\frac{h_{0}}{k_{0}}, \frac{h}{k}, \frac{h_{1}}{k_{1}}$ are three consecutive terms of the Farey sequence of order $N$. Note that each $\theta$ satisfies $\frac{1}{2 k N} \leq \theta \leq \frac{1}{k N}$.

The following transformation formula of the Dedekind eta function plays an important role in the circle method. For $\gamma=\left(\begin{array}{ll}a & b \\ c & d\end{array}\right) \in \mathrm{SL}_{2}(\mathbb{Z})$, we have

$$
\eta(\gamma z)=e^{-\pi i s(d, c)} e^{\frac{\pi i(a+d)}{12 c}} \sqrt{-i(c z+d)} \eta(z)
$$

where $s(d, c)$ is the Dedekind sum defined by $s(d, c)=\sum_{r=1}^{c-1}\left(\frac{r}{c}-\left[\frac{r}{c}\right]-\frac{1}{2}\right)\left(\frac{d r}{c}-\left[\frac{d r}{c}\right]-\frac{1}{2}\right)$.

We define $\omega_{h, k}=e^{\pi i s(h, k)}$. From Equation (10) and $E_{4}(z) \in M_{4}\left(\Gamma_{0}(1)\right)$, we derive the transformation property of $G(z)=q g_{1}(z)$. 
Proposition 1 Let $h, k$ be integers such that $k>0$ and $(h, k)=1$. For each $d$, let $h_{d}$ be an integer such that $d h h_{d} \equiv-(d, k)(\bmod k)$. Then,

$$
\begin{aligned}
G(\exp (2 \pi i \tau)) & =\omega(h, k) \exp \left(\frac{\pi}{12 k}\left(-o(G, k) \frac{1}{z}-24 z\right)\right) z^{-3 / 2} \sqrt{\frac{(4, k)^{2}}{8(2, k)}} \\
& \times \frac{F\left(\exp \left(\frac{(2, k) h_{2}}{k}+i \frac{(2, k)^{2}}{2 k z}\right)\right) F^{6}\left(\exp \left(\frac{(4, k) h_{4}}{k}+i \frac{(4, k)^{2}}{4 k z}\right)\right)}{F^{2}\left(\exp \left(\frac{h_{1}}{k}+i \frac{1}{k z}\right)\right)} \\
& \times E_{4}\left(\exp \left(\frac{(4, k) h_{4}}{k}+i \frac{(4, k)^{2}}{4 k z}\right)\right)
\end{aligned}
$$

where $F\left(e^{2 \pi i z}\right)=\frac{q^{1 / 24}}{(\eta(z))}, \omega(h, k)=\exp \left(\pi i\left(-2 s(h, k)+s\left(\frac{2 h}{(2, k)}, \frac{k}{(2, k)}\right)+6 s\left(\frac{4 h}{(4, k)}, \frac{k}{(4, k)}\right)\right)\right)$ and $o(G, k)=0,-6,-24$ according to $(k, 4)=1,2,4$.

The following lemma and two estimations are crucial when we determine the main term and the bound of the error term.

Lemma 1 Let us define

$$
I:=\int_{\xi h, k} z^{-\frac{3}{2}} \exp \left(\frac{\pi}{12 k}\left(\frac{b}{z}-c z\right)\right) e^{2 \pi n \rho} e^{-2 \pi i n \varphi} d \varphi
$$

where $b$ is a positive integer. Then,

$$
I=\sqrt{\frac{48}{k b}} \sinh \left(\frac{\pi}{k} \sqrt{\frac{2 b}{3}\left(n-\frac{c}{24}\right)}\right)+E(I)
$$

where $|E(I)| \leq e^{\frac{\pi b}{3}}(2 N)^{3 / 2} \frac{e^{2 \pi\left(n-\frac{c}{24}\right) \rho}}{\pi\left(n-\frac{c}{24}\right)}$.

Let $P(q)=\prod_{n=0}^{\infty}\left(1-q^{n}\right)^{-1}=\sum_{n=0}^{\infty} p(n) q^{n}$ be the generating function for $p(n)$. We use the following estimation given by Chan ([14] [Equation (3.19)]):

$$
\sum_{n=0}^{\infty} p(n) e^{-2 \pi y n} \leq \exp \left(\frac{e^{-2 \pi y}}{\left(1-e^{-2 \pi y}\right)^{2}}\right)=: U_{p}(y)
$$

where $y$ is a positive real number. By using a trivial bound $\sigma_{3}(n) \leq n^{4}$, we also see that

$$
1+240 \sum_{n=1}^{\infty} \sigma_{3}(n) e^{-2 \pi y n} \leq 1+240 \sum_{n=1}^{\infty} n^{4} e^{-2 \pi y n} \leq 1+5760 \frac{e^{-2 \pi y}}{\left(1-e^{-2 \pi y}\right)^{5}}=: U_{E}(y)
$$

Now we are ready to estimate $B_{1}(1, d)$. As the procedure of the proof is similar to that of [14] and the detailed calculation for bounding error term is tedious, we give only outlines. By Equation (7) and $G(z)=q g_{1}(z)$, we see that

$$
\begin{aligned}
B_{1}(1, n-1) & =\left(\sum_{\substack{1 \leq k \leq N \\
(k, 4)=1}}+\sum_{\substack{1 \leq k \leq N \\
(k, 4)=2}}+\sum_{\substack{1 \leq k \leq N \\
4 \mid \bar{k}}}\right) \sum_{\substack{0 \leq h \leq k \\
(h, k)=1}} e^{\frac{-2 \pi i n h}{k}} \int_{\xi_{h, k}} G(\tau) e^{2 \pi n \rho} e^{-2 \pi i n \varphi} d \varphi \\
& =: S_{1}(A)+S_{2}(A)+S_{3}(A)
\end{aligned}
$$


where $A$ is the integrand.

For the first sum $S_{1}(A)$, by using the transformation formula (Proposition 1), Equations (14) and (15), and the fact that $\frac{2 \pi}{k} \operatorname{Re} \frac{1}{z} \geq \pi$, we obtain

$$
|A| \leq \frac{1}{\sqrt{8}} U_{p}\left(\frac{1}{8}\right)^{7} U_{E}\left(\frac{1}{8}\right)|z|^{-\frac{3}{2}} e^{2 \pi(n-1) \rho}
$$

Here, we have used the fact that $\prod_{k=1}^{\infty} \frac{\left(1-q^{2 k}\right)^{2}}{1-q^{k}}$ is a generating function for the number of 2-core partitions of $n$, and therefore $t(n) \leq p_{7}(n)$ for all positive integers $n$, where

$$
\sum_{n=0}^{\infty} t(n) q^{n}=\prod_{k=1}^{\infty} \frac{\left(1-q^{4 k}\right)^{2}}{\left(1-q^{k}\right)^{6}\left(1-q^{2 n}\right)} \quad \text { and } \quad \sum_{n=0}^{\infty} p_{7}(n) q^{n}=\prod_{k=1}^{\infty} \frac{1}{\left(1-q^{k}\right)^{7}}
$$

Since the length of the integration is less than $\frac{2}{k N}$ and $|z|^{-3 / 2} \leq k^{-3 / 2} N^{3}$, we see that

$$
\left|S_{1}(A)\right| \leq \frac{1}{\sqrt{2}} \zeta(3 / 2) U_{p}\left(\frac{1}{8}\right)^{7} U_{E}\left(\frac{1}{8}\right) e^{2 \pi(n-1) \rho} N^{2}
$$

where $\zeta(s)$ is the Riemann zeta function.

We now turn to the estimation of $S_{3}$. By applying Proposition 1, we observe that

$$
\begin{aligned}
S_{3} & =\sum_{\substack{1 \leq k \leq N \\
4 \mid k}} \sum_{\substack{0 \leq h \leq k \\
(h, k)=1}} e^{\frac{-2 \pi i n h}{k}} \int_{\xi_{h, k}} \omega(h, k) \sqrt{2} z^{-\frac{3}{2}} \exp \left(\frac{24 \pi}{12 k}\left(z-\frac{1}{z}\right)\right) e^{2 \pi n \rho} e^{-2 \pi i n \varphi} d \varphi \\
& +\sum_{\substack{1 \leq k \leq N \\
4 \mid \bar{k}}} \sum_{\substack{0 \leq h \leq k \\
(h, k)=1}} e^{\frac{-2 \pi i n h}{k}} \int_{\xi_{h, k}} \omega(h, k) \sqrt{2}\left(G\left(\tau^{\prime}\right)-1\right) z^{-\frac{3}{2}} \exp \left(\frac{24 \pi}{12 k}\left(z-\frac{1}{z}\right)\right) e^{2 \pi n \rho} e^{-2 \pi i n \varphi} d \varphi \\
& =: T_{31}+T_{32}
\end{aligned}
$$

Estimation of $T_{32}$ is very similar to that of $S_{1}$ :

$$
\left|T_{32}\right| \leq 2 \sqrt{2} e^{-\pi} \zeta(3 / 2)\left(U_{p}\left(\frac{1}{2}\right)^{9} U_{E}(2)-1\right) e^{2 \pi(n-1) \rho} N^{2}
$$

From Lemma 1, we see that

$$
T_{31}=\sum_{\substack{1 \leq k \leq N \\ 4 \mid k}} \sum_{\substack{0 \leq h \leq k \\(h, k)=1}} e^{\frac{-2 \pi i n h}{k}} \omega(h, k) \sqrt{\frac{2}{k}} \sinh \left(\frac{4 \pi}{k} \sqrt{n-1}\right)+E_{1, n}
$$

where

$$
\left|E_{1, n}\right| \leq \sqrt{2} e^{8 \pi}(2 N)^{3 / 2} \frac{e^{2 \pi(n-1) \rho}}{\pi(n-1)}
$$

For $S_{2}$, by decomposing as $S_{3}$ case, we see that

$$
\left|T_{22}\right| \leq e^{-\pi / 4} \zeta(3 / 2)\left(U_{p}\left(\frac{1}{2}\right)^{5} U_{E}\left(\frac{1}{2}\right)-1\right) e^{2 \pi(n-1) \rho} N^{2}
$$


and

$$
T_{21}=\sum_{\substack{1 \leq k \leq N \\(k, 4)=2}} \sum_{\substack{0 \leq h \leq k \\(h, k)=1}} e^{\frac{-2 \pi i n h}{k}} \omega(h, k) \sqrt{\frac{2}{k}} \sinh \left(\frac{2 \pi}{k} \sqrt{n-1}\right)+E_{2, n}
$$

where

$$
\left|E_{2, n}\right| \leq \sqrt{2} e^{2 \pi}(2 N)^{3 / 2} \frac{e^{2 \pi(n-1) \rho}}{\pi(n-1)}
$$

In summary, we have shown that

$$
B_{1}(1, n) \sim \frac{1}{2} C(n+1) \exp (\pi \sqrt{n})
$$

where

$$
\sqrt{2} C(n):=\sqrt{2} e^{-\pi n i} \omega(1,2)+e^{-\pi n i / 2} \omega(1,4)+e^{-3 \pi n i / 2} \omega(3,4)
$$

Therefore, the sign of $B_{1}(1, n)$ is determined by that of $C(n+1)$ for sufficiently large $n$. We note that $C(n)$ is of period 4 with

$$
C(1)=-2, \quad C(2)=C(3)=0, \quad C(4)=2
$$

Note that $g_{1}(z)$ is in Kohnen's plus space, and hence $n$-th Fourier coefficient vanishes if $n \equiv 1$ or 2 (mod 4$)$. After a simple, but lengthy calculation, we find that error terms are dominated by

$$
\frac{\sqrt{2}}{2}(2 \pi n)^{3 / 2} \exp \left(\frac{\pi}{3} \sqrt{n}\right)
$$

for all integers $n>875$. By checking the first 874 terms, we observe that

$$
\left|B_{1}(1, n)-\frac{1}{2} C(n+1) \exp (\pi \sqrt{n})\right| \leq \frac{1}{2}(2 \pi n)^{3 / 2} \exp \left(\frac{\pi}{3} \sqrt{n}\right)
$$

for all positive integers $n$. Since $\exp (\pi \sqrt{n})>(2 \pi n)^{3 / 2} \exp \left(\frac{\pi}{3} \sqrt{n}\right)$ for all integers $n>1$, we find that the sign of $B_{1}(1, n)$ is determined by $C(n+1)$ for all positive integers $n$ by checking the first term.

Now we turn to the investigation on the image of $g_{1}(z)$ under Hecke operators. For a prime $p$, we define the Hecke operator $T\left(p^{2}\right)$ on $M_{\frac{3}{2}}^{!,+}\left(\Gamma_{0}(4)\right)$ by

$$
\left(\left.f\right|_{\frac{3}{2}} T\left(p^{2}\right)\right)(z):=\sum_{\substack{n \in \mathbb{Z} \\ n \equiv 0,3(\bmod 4)}}\left(c\left(p^{2} n\right)+\left(\frac{-n}{p}\right) c(n)+p c\left(n / p^{2}\right)\right) q^{n}
$$

where $f(z)=\sum_{n \in \mathbb{Z}} c(n) q^{n}$ (see [18][Proposition 2]). For a positive integer $m$, the Hecke operator $T\left(m^{2}\right)$ is defined by the following recursive relation (see [19][Theorem 1])

$$
T\left(p^{2 l+2}\right)=T\left(p^{2}\right) T\left(p^{2 l}\right)-p T\left(p^{2 l-2}\right)
$$

and the multiplicity property that $T\left(m^{2} m^{\prime 2}\right)=T\left(m^{2}\right) T\left(m^{\prime 2}\right)$ whenever $\left(m, m^{\prime}\right)=1$. We derive that

$$
\left(\left.f\right|_{\frac{3}{2}} T\left(m^{2}\right)\right)(z)=\sum_{\substack{n \in \mathbb{Z} \\ n \equiv 0,3(\bmod 4)}}\left(\sum_{d \mid m^{2}} b(d) c\left(m^{2} n / d^{2}\right)\right) q^{n}
$$


where $b(d) \in \mathbb{R}$ depending on $d$. After some computations we find that $|b(d)| \leq m^{2}$ for all $d$. Recall that, for all positive integers $n$, we have obtained

$$
\left|B_{1}(1, n)\right|<A(n)+B(n)
$$

and

$$
B(n)<\frac{3}{2} A(n)
$$

where $A(n)=\frac{1}{2} C(n+1) \exp (\pi \sqrt{n})$ and $B(n)=\frac{\sqrt{2}}{2}(2 \pi n)^{\frac{3}{2}} \exp \left(\frac{\pi}{3} \sqrt{n}\right)$. Therefore, we arrive at

$$
\begin{aligned}
\left|B_{m}(1, n)-A\left(m^{2} n\right)\right| & =\left|\sum_{d \mid m^{2}} b(d) B_{1}\left(1, m^{2} n / d^{2}\right)-A\left(m^{2} n\right)\right| \\
& <B\left(m^{2} n\right)+\sum_{d \mid m^{2} \text { and } d \neq 1} \frac{5}{2} b(d) A\left(m^{2} n / d^{2}\right) \\
& <B\left(m^{2} n\right)+5 m^{3} A\left(m^{2} n / 4\right)
\end{aligned}
$$

where the last inequality comes from the fact that $\sum_{d \mid m^{2}} 1 \leq 2 m$ for all positive integers $m$. Hence, we see that

$$
\left|B_{m}(1, n)-A\left(m^{2} n\right)\right|<B\left(m^{2} n\right)+5\left(m^{2} n\right)^{2} A\left(m^{2} n / 4\right)
$$

and observe that $A(k)>B(k)+5 k^{2} A(k / 4)$ for all integers $k>27$. Therefore, the sign of $B_{m}(1, n)$ is determined by $B_{1}\left(1, m^{2} n\right)$ if $m^{2} n>24$ and we can easily verify that the same is true for $m^{2} n \leq 27$.

\section{Acknowledgements}

The first two named authors would like to thank KIAS for support throughout the associate membership program. Dohoon Choi was supported by Basic Science Research Program through the National Research Foundation of Korea (NRF) funded by the Ministry of Education, Science and Technology (NRF2010-0022180). Byungchan Kim was supported by Basic Science Research Program through the National Research Foundation of Korea (NRF) funded by the Ministry of Education, Science and Technology (NRF2011-0009199).

\section{Conflicts of Interest}

The authors declare no conflict of interest.

\section{References}

1. Zagier, D. Traces of Singular Moduli. In Motives, Polylogarithms and Hodge Theory; Int. Press Lect. Ser. 3; International Press: Somerville, MA, USA, 2002; pp. 209-244.

2. Ahlgren, S. Hecke relations for traces of singular moduli. Bull. Lond. Math. Soc. 2012, 44, 99-105.

3. Ahlgren, S.; Ono, K. Arithmetic of singular moduli and class polynomials. Compos. Math. 2005, $141,293-312$. 
4. Guerzhoy, P. Some congruences for traces of singular moduli. J. Number Theory 2007, 122, 151-156.

5. Bringmann, K.; Ono, K. Arithmetic properties of coefficients of half-integral weight Maass-Poincaré series. Math. Ann. 2007, 337, 591-612.

6. Folsom, A.; Ono, K. Duality involving the mock theta function $f(q)$. J. Lond. Math. Soc. 2008, 77, 320-334.

7. Bruinier, J.H.; Funke, J. Traces of CM values of modular functions. J. Reine Angew. Math. 2006, 594, 1-33.

8. Bruinier, J.H.; Jenkins, P.; Ono, K. Hilbert class polynomials and traces of singular moduli. Math. Ann. 2006, 344, 373-393.

9. Choi, D.; Jeon, D.; Kang, S.; Kim, C. Traces of singular moduli of arbitrary level modular functions. Int. Math. Res. Not. 2007, doi:10.1093/imrn/rnm110.

10. Bruinier, J.H.; Kohnen, W. Sign Changes of Coefficients of Half Integral Weight Modular Forms. In Modular Forms on Schiermonnikoog; Cambridge University Press: Cambridge, UK, 2008; pp. 57-66.

11. Choie, Y.; Kohnen, W. The first sign change of Fourier coefficients of cusp forms. Am. J. Math. 2009, 131, 517-543.

12. Knopp, M.I.; Kohnen, W.; Pribitkin, W. On the signs of Fourier coefficients of cusp forms. Ramanujan J. 2003, 7, 269-277.

13. Kohnen, W.; Sengupta, J. On the first sign change of Hecke eigenvalues of newforms. Math. Z. 2006, 254, 173-184.

14. Chan, O.-Y. Some asymptotics for cranks. Acta Arith. 2005, 120, 107-143.

15. Kane, D.M. Resolution of a conjecture of Andrews and Lewis involving cranks of partitions. Proc. Am. Math. Soc. 2004, 132, 2247-2256.

16. Kim, B. Periodicity of signs of Fourier coefficients of eta quotients. J. Math. Anal. Appl. 2012, 385, 998-1004.

17. Andrews, G.E. The Theory of Partitions; Addison-Wesley: Reading, MA, USA, 1976; reissued by Cambridge University Press: Cambridge, UK, 1998.

18. Kohnen, W. Modular forms of half-integral weight on $\Gamma_{0}(4)$. Math. Ann. 1980, 248, 249-266.

19. Purkait, S. Hecke operators in half-integral weight. 2012, arXiv:1208.4326v1.

(c) 2013 by the authors; licensee MDPI, Basel, Switzerland. This article is an open access article distributed under the terms and conditions of the Creative Commons Attribution license (http://creativecommons.org/licenses/by/3.0/). 